\title{
ANÁLISE DE DADOS DE PERCEPÇÃO E PRODUÇÃO DO ESPANHOL/LÍNGUA ESTRANGEIRA POR FALANTES DO PORTUGUÊS BRASILEIRO EM ZONA DE FRONTEIRA ${ }^{1}$
} ANALYSIS OF SPANISH AS A FOREIGN LANGUAGE PERCEPTION AND PRODUCTION DATA BY SPEAKERS OF BRAZILIAN PORTUGUESE IN BORDER ZONES

\author{
Giane Rodrigues dos Santos ${ }^{2}$
}

Resumo: Este trabalho apresenta parte dos resultados de Santos (2014), que investigou a percepção e a produção das vogais médias do espanhol por falantes do português brasileiro (PB). Visto que no espanhol não há as vogais médias-baixas /E/ e /o/ como ocorre no PB (MARTÍNEZ CELDRÁN; FERNÁNDEZ PLANAS, 2007), essa diferença pode ser considerada um dos motivos pelos quais os falantes do $P B$ pronunciam as vogais médias do espanhol [e] e [o] como as médias-baixas do $P B$ [E] e [o], respectivamente (BAZZAN, 2005; MIGNONI, 1999). Assim, 40 alunos de Cursos de Licenciaturas em Espanhol foram submetidos a um teste de percepção e a um teste de produção. Quanto à percepção das vogais-alvo, não foram encontradas diferenças significativas entre a zona de fronteira direta e indireta. Quanto à produção, os dados foram analisados acusticamente com o software Praat e revelam que, em relação às propriedades vocálicas (duração, F1 e F2), também não foram encontradas diferenças significativas entre os grupos analisados.

Palavras-chave: vogais médias; espanhol L2; zona de fronteira.

Abstract: This paper reports part of the results by Santos (2014), who investigated the perception and production of the Spanish mid vowels by speakers of Brazilian Portuguese (BP). Considering that Spanish does not have the low mid vowels / $\varepsilon /$ or $/ \mathrm{J}$, as does BP (MARTÍNEZ CELDRÁN; FERNÁNDEZ PLANAS, 2007), this difference may be considered one of the reasons BP speakers pronounce the Spanish mid vowels [e] and [o] as the BP low mid vowels [E] and [o], respectively (BAZZAN, 2005; MIGNONI, 1999). Thus, 40 students of the undergraduate "Licenciaturas em Espanhol" course took both

1 Estudo realizado com bolsa PDSE/CAPES/UCPEL.

2 Doutora em Letras - Linguística Aplicada pela UCPel. 
a perception test and a production test. As regards perception of the target vowels, no significant differences were found between direct and indirect border zones. As regards production, the data were acoustically analyzed with the software Praat and reveal that, in terms of vowel properties (duration, F1 and F2), no significant differences were found among the observed groups. Keywords: mid vowels; Spanish L2; border zones.

\section{Introdução}

Alguns estudos precursores que buscaram investigar as dificuldades dos falantes do português brasileiro (PB) com aspectos fonéticos do espanhol como língua estrangeira (E/LE), baseados em análises contrastivas, evidenciaram várias influências da língua materna (L1), como, por exemplo, o fenômeno de abertura das vogais médias, perda de timbre, nasalização, vocalização da consoante lateral, ditongação e presença de epêntese (HOYOS-ANDRADE, 1978; MASIP, 1995). Especificamente, em se tratando de dificuldades com as vogais médias /e/ e /o/ do E/LE por falantes do $\mathrm{PB}$, há dois estudos realizados no sul do Brasil com estudantes de Cursos de Licenciaturas em Espanhol, que são os estudos de Mignoni (1999) e Bazzan (2005). Esses trabalhos investigaram a produção das vogais médias do espanhol /e/ e /o/ por falantes do PB, com análises baseadas em teorias fonológicas. Tais estudos evidenciaram a tendência dos falantes do PB em empregar vogais médias-baixas mesmo em contextos em que nativos do espanhol (E/L1) empregariam vogais médias-altas. Porém, os autores não fizeram análises acústicas e estatísticas dos dados, nem os relacionaram a dados de percepção.

Conforme Câmara Jr. (2007 [1970]), o sistema vocálico do português brasileiro $(\mathrm{PB})$ possui sete vogais orais $(/ \mathrm{i}, \mathrm{e}, \varepsilon, \mathrm{a}, \mathrm{\jmath}, \mathrm{o}, \mathrm{u} /)$ em posição tônica. Em posição pretônica, há cinco vogais (/i, e, a, o, u/), não se verificando a realização das vogais médias-baixas $(/ \varepsilon, \diamond /)$, exceto em alguns dialetos. Já em posição postônica não final, o sistema sofre redução por eliminação da oposição entre /o/ e /u/ e em posição pós-tônica final, e o quadro vocálico do PB fica reduzido a três fonemas vocálicos $(/ \mathrm{a}, \mathrm{i}, \mathrm{u} /)$, porque as vogais médias-altas /e/ e /o/ ficam reduzidas, respectivamente, a /i/ e /u/, como em "pele" ['pe.li] e dedo ['de.du]. Quanto ao espanhol, há apenas cinco fonemas vocálicos: /i, e, a, o, u/. Não há na língua espanhola vogais médias-baixas com distinção fonológica, embora foneticamente haja realizações com maior ou menor grau de abertura vocálica (MARTÍNEZ CELDRÁN; 
FERNÁNDEZ PLANAS, 2007). Essa diferença entre os sistemas vocálicos do espanhol e do $\mathrm{PB}$ quanto à não existência das vogais médias-baixas $(/ \varepsilon$, ऽ/) no espanhol, do ponto de vista linguístico, pode ser um dos motivos pelos quais os falantes do PB pronunciam os sons do espanhol com sotaque de sua língua materna (L1).

Considerando-se as diferenças entre os sistemas vocálicos do PB e do espanhol, cabe salientar a relevância que tem o ensino de pronúncia nas salas de aula de língua estrangeira (L2) como parte das habilidades comunicativas exigidas na formação, tanto por parte dos professores, como dos próprios alunos, que futuramente estarão atuando como professores de línguas.

Estudos de percepção de sons de L2 são relativamente recentes no Brasil (e.g., RESTREPO, 2013; RAUBER, 2010; KLUGE, 2004, 2009; SILVEIRA, 2004; PASCA, 2003; KOERICH, 2002) e possuem diferentes objetos de estudo (vogais, consoantes), em diferentes sistemas de L2 (espanhol, francês, inglês), com metodologias e resultados diversificados. Considerando-se a falta de pesquisas que inter-relacionem resultados de percepção e produção de sons, o objetivo geral deste trabalho é investigar a percepção e a produção das vogais médias ([e, o]) do espanhol como L2 (E/L2) por falantes do PB em Cursos de Letras - Habilitação em Espanhol como Língua Estrangeira, em semestres finais de estudo do E/L2.

Os objetivos específicos deste trabalho são: (i) realizar medições acústicas das vogais médias do E/L2 produzidas por falantes do PB, a fim de obter valores de formantes em diferentes contextos de aprendizagem: zona de fronteira direta versus zona de fronteira indireta, visto que a maior ou a menor proximidade com a fronteira Brasil - Uruguai pode ter influências na produção das falantes de E/L2; (ii) investigar a percepção das vogais médias no contexto zona de fronteira, a fim de comparar o desempenho dos estudantes de fronteira direta com os de fronteira indireta; (iii) correlacionar o desempenho dos alunos quanto à percepção e à produção das vogais médias do espanhol, considerando a zona de fronteira.

Com base em estudos preliminares e na revisão de literatura, levantam-se as seguintes questões: (I) Q1- Como será o desempenho dos falantes do E/L2 nos grupos fronteira direta e fronteira indireta, quanto à percepção das vogais-alvo?; (II) Q1- As medições formânticas e de duração das vogais médias do espanhol produzidas por falantes do E/L2 apresentarão diferenças em relação à zona de fronteira direta e fronteira indireta?

Com vistas a responder às questões norteadoras, este trabalho está organizado da seguinte maneira: primeiramente, serão apresentados estudos de percepção e produção de sons de L2 e considerações sobre a zona de 
fronteira. Na sequência, será apresentada a metodologia utilizada para a coleta e a análise dos dados pesquisados, e, finalmente, serão apresentadas as análises dos dados de percepção e produção, bem como as considerações finais sobre este estudo.

\section{Fundamentação teórica}

Esta seção apresenta brevemente os principais pressupostos dos modelos mais usados em estudos de percepção de sons de L2 e reporta resultados de estudos que abordaram a relação entre percepção e produção de sons em L2.

\subsection{Percepção e produção de sons em L2}

Estudos têm demonstrado que o sistema vocálico da L1, por exemplo, tem influência direta na produção e na percepção das vogais de uma L2, principalmente quando se começa a aprender essa L2 após a infância (FLEGE, 1987, 1995; FLEGE; BOHN; JANG, 1997; MAJOR, 1987; RAUBER, 2010; ROCHET, 1995). Alguns estudos que investigaram a relação entre a percepção e a produção de vogais de uma L2 encontraram uma correlação moderada (BRADLOW, 1996; FLEGE et al., 1997; FLEGE; MACKAY; MEADOR, 1999; RAUBER, 2010; ROCHET, 1995). Os resultados desses estudos corroboram a hipótese de que uma pronúncia não nativa de sons da L2 pode ser motivada por uma percepção incorreta desses sons, já que os sons da L2 que não foram bem percebidos também foram os produzidos com valores mais distantes aos das médias dos valores produzidos por falantes nativos.

Atualmente, os modelos mais influentes que explicam a percepção de sons não-nativos, aplicados à Aquisição Fonética de L2, são o Modelo de Aprendizagem de Fala (Speech Learning Model-SLM) de Flege (1995) e o Modelo de Assimilação Perceptual (Perceptual Assimilation Model-PAM-L2) de Best e Tyler (2007).

Segundo Flege (1995), a premissa básica do SLM é de que categorias fonêmicas adquiridas no início da vida permanecem maleáveis até certo ponto e que indivíduos podem tanto desenvolver categorias já existentes na L1, quanto desenvolver categorias da L2. Ou seja, os processos que os falantes de uma língua utilizam para estabelecer o sistema de L1 permanecem intactos durante toda a vida, podendo ser acessados durante a aprendizagem de uma L2. Nesse modelo, os sistemas de sons da L1 e da L2 são 
compartilhados no mesmo espaço fonológico do falante, o que significa que os sistemas interagem entre si. Assim, postula-se que pode ser difícil para uma categoria da L2, cujas características acústicas difiram das categorias da L1, alterar padrões já estabelecidos na L1, sendo classificada como uma categoria já existente na L1.

Por outro lado, segundo Best e Tyler (2007, p. 15), o PAM foi desenvolvido especificamente para explicar a percepção de fala por ouvintes não-nativos "naïve" (ingênuos) ou inexperientes, ou seja, ouvintes que desconheciam as línguas sendo testadas, enquanto que o SLM foi elaborado para explicar a percepção da fala de L2 por aprendizes dessa L2. Exatamente devido às aplicações indevidas do PAM a situações envolvendo uma L2, Best e Tyler (2007) buscaram explorar as semelhanças e diferenças entre o SLM e o PAM para a ampliação do PAM também para L2. Best e Tyler alertam que os dois modelos divergem em alguns pontos e convergem em outros. Por exemplo, ambos os modelos compartilham a noção de que os processos de aprendizagem perceptual permanecem intactos ao longo da vida; e divergem quanto à base teórica e às unidades de análise.

Segundo Strange (2007), teorias e modelos de percepção e produção de L2 têm se empenhado em explicar as dificuldades dos aprendizes de L2 em relação ao aprendizado de alguns segmentos e contrastes fonéticos não-nativos. Em cada teoria, o conceito de semelhanças fonéticas entre línguas (L1/L2) é empregado para fazer referência ao fato de que alguns contrastes não-nativos são mais difíceis de diferenciar perceptualmente (e produzir autenticamente) do que outros, tanto em fase inicial como depois de anos de experiência com aprendizagem de L2.

Nos estudos de aprendizagem de uma L2, o fator idade é referenciado tanto em pesquisas de percepção como de produção, como, por exemplo, nos estudos relatados em Piske et al. (2001). Outras pesquisas evidenciam forte influência da experiência na ou contato com a produção acurada de sons de uma L2 (FLEGE, 1997). De acordo com Flege, a maior experiência com a L2 pode gradualmente aumentar a sensibilidade perceptual de diferenças fonéticas entre a L1 e a L2, ou melhorar a percepção de diferenças entre os sons de L2 que não são contrastantes na L1. Isso pode aumentar a probabilidade de novas categorias serem estabelecidas para sons encontrados na L2 e inexistentes na L1, o que, por sua vez, pode resultar em maior precisão na produção da L2.

Segundo Flege (1997, p. 438), alguns pesquisadores acreditam que a percepção de vogais e consoantes permanece de certa forma maleável, mesmo na idade adulta. Assim, embora a acurácia da produção de L2 pos- 
sa ser limitada por fatores perceptuais, a capacidade de aprender novas formas de fala permanece intacta ao longo da vida (FLEGE, 1988, 1995). Esses pontos de vista implicam que, dado input suficiente de pronúncia ideal e considerando-se a ausência de limitações de base perceptuais, adultos podem eventualmente aprender a produzir certas vogais da L2 com acurácia nativa. Iniciantes adultos interpretam tipicamente vogais da L2 como vogais semelhantes às vogais da $\mathrm{L} 1 \mathrm{e}$, consequentemente, produzem vogais da L2 com características acústicas da L1. Investigar se vogais de L1 e L2 são perceptualmente relacionadas umas com as outras permite inferir como aprendizes adultos inexperientes produzirão vogais da L2. Entretanto, se processos de percepção de fala permanecem maleáveis (BEST; STRANGE, 1992) ou se aprendizes de L2 estabelecem categorias fonéticas novas para certas vogais de L2 (FLEGE, 1995), então a relação percebida entre vogais da L1 e da L2 pode mudar durante a aquisição da L2. Assim, mudanças na percepção podem por sua vez produzir mudanças na produção vocálica (FLEGE, 1997, p. 440).

Os principais estudos sobre percepção de vogais dizem respeito à aquisição dos segmentos vocálicos do inglês (americano) por diversos falantes não-nativos, conforme verificado em Bohn e Flege (1992), Bohn (1995), Flege, MacKay e Meaddor (1999), Escudero (2000, 2001), Morrison (2002). Pesquisas atuais abordam a percepção juntamente com a produção, como, por exemplo, as pesquisas sobre as vogais de diferentes sistemas de L2 por falantes do PB (RAUBER, 2006; NOBRE-OLIVEIRA, 2007; RAUBER; RATO; SILVA, 2010; FLORES; RAUBER, 2010; RESTREPO, 2013).

Um dos estudos pioneiros sobre percepção de vogais foi o de Fox (1982), que testou falantes de inglês americano através de uma escala perceptual vocálica e tarefas de produção. $\mathrm{O}$ autor encontrou diferenças consistentes de percepção entre os ouvintes, diferenças essas que correspondiam às diferenças encontradas na produção. Ele mostrou que as propriedades acústicas de vogais produzidas predizem suas medidas de percepção ao longo da mesma dimensão acústica. Assim, Fox defende uma ligação direta entre percepção e produção.

Quantos aos estudos mais recentes que investigaram a relação entre a percepção e produção de vogais de L2 por falantes do $\mathrm{PB}$, pode-se citar os trabalhos de Rauber et al. (2010) e Restrepo (2013). Rauber et al. (2010) examinaram a percepção e a produção de vogais anteriores do inglês por dez falantes do mandarim. Os resultados revelaram que o desempenho perceptual é superior ao produtivo, e que a percepção e a produção estão relacionadas, já que as taxas de identificação mais elevadas no teste de 
percepção correspondiam a melhores resultados em termos de produção, e as taxas de identificação mais baixas, por sua vez, correspondiam a uma produção menos precisa.

O estudo de Restrepo (2013) investigou a percepção e a produção das vogais anteriores médias arredondadas do francês [ø] e [œ] por seis informantes femininas falantes nativas do PB aprendizes de francês. Os resultados demonstraram que o tempo de aprendizado (ou experiência) com a língua estrangeira melhora a percepção que as estudantes brasileiras têm das vogais [ø] e [œ], e que a melhoria da percepção é acompanhada de uma melhoria na qualidade da produção das mesmas vogais.

Quanto à percepção de vogais do espanhol por falantes do PB, o estudo de Pasca (2003) é pioneiro na região sul do Brasil. A autora investigou a percepção da nasalidade da vogal oral /a/ por 74 estudantes de língua portuguesa da Universidade Federal do Rio Grande do Sul, em Porto Alegre. Segundo Pasca (2003), os estudantes tiveram ligeiramente mais facilidade em perceber a vogal-alvo em palavras isoladas, com o percentual de $81 \%$, do que em palavras em texto, com a taxa de $78 \%$ (não foram aplicados testes estatísticos inferenciais). Já entre as palavras cognatas e não cognatas, não houve diferença ( $81 \%$ a $81 \%$, respectivamente). A autora ainda conclui que a distinção entre oralidade e nasalidade da vogal /a/ é mais perceptível em sílaba tônica, evidenciando a relevância da tonicidade para a percepção.

Resumidamente, apesar da maioria das pesquisas empíricas anteriores suportarem a existência da simetria entre percepção e produção, essa relação permanece desafiadora aos pesquisadores, principalmente aos modelos de aquisição de fonética/fonologia de L2, com repercussões diretas e práticas nas metodologias utilizadas nas pesquisas.

Na próxima seção serão descritos os procedimentos metodológicos adotados em todas as etapas de coleta e análise de dados neste estudo, que objetivou investigar a percepção e a produção de vogais médias do espanhol por falantes do PB.

\section{Metodologia}

Para esta pesquisa, que analisa e relaciona dados de percepção com dados de produção de fala, vários cuidados metodológicos foram seguidos de acordo com pesquisas anteriores, como, por exemplo, Rauber (2010) e Escudero et al. (2009), cuidados esses que serão descritos brevemente nas subseções seguintes. 


\subsection{Participantes}

As participantes do estudo são 40 falantes femininas de PB como língua materna e falantes de espanhol L2 (E/L2), todas alunas de Cursos de Licenciatura em Letras-Habilitação em Espanhol, nas cidades de Pelotas, Jaguarão, Santana do Livramento e Rio Grande, conforme a Figura 1.

\begin{tabular}{|c|c|c|c|}
\hline Cidade & Modalidade & Zona de Fronteira & Participantes \\
\hline Pelotas & Presencial & fronteira indireta & 8 \\
\hline Pelotas & Distância & fronteira indireta & 8 \\
\hline Jaguarão & Presencial & fronteira direta & 8 \\
\hline Santana do Livramento & Distância & fronteira direta & 8 \\
\hline Rio Grande & Presencial & fronteira indireta & 8 \\
\hline Total & -- & -- & 40 \\
\hline
\end{tabular}

Figura 1- Participantes da pesquisa (E/L2)

Também foram coletados dados em dois grupos de controle, com oito informantes femininas em cada grupo: 1) na cidade de Rio Grande (RS) para amostra do PB como L1 (PB/L1); e 2) na cidade de Montevidéu (Uruguai) para a amostra do espanhol como L1 (E/L1), descritos detalhadamente em Santos (2014).

\section{$3.2 \mathrm{O}$ contexto zona de fronteira}

De acordo com documentos do Banco de Dados Sociolinguísticos-Pampa, as cidades de Pelotas e Rio Grande fazem parte da chamada "zona de fronteira”, por estarem localizadas dentro dos $200 \mathrm{~km}$ de faixa territorial brasileira que faz divisa com o Uruguai (ESPIGA, 2001). Para poder diferenciar as quatro cidades (Jaguarão, Pelotas, Rio Grande, Santana do Livramento) em que seriam coletados os dados, buscou-se uma nomenclatura a fim de diferenciá-las. Assim, optou-se por usar os termos de fronteira direta para cidades gêmeas (mesmo que separadas por rios, pontes), como, por exemplo, para as cidades de Jaguarão e Santana do Livramento, e fronteira indireta para as cidades de Pelotas e Rio Grande.

Optou-se por estudar quatro cidades, Jaguarão, Pelotas, Rio Grande e Santana do Livramento (FIGURA 2), devido à experiência da autora com alunos nestas cidades, evidenciando que os alunos de fronteira direta possuíam um maior contato diário com a língua espanhola, ou seja, maior frequência e maior input em língua espanhola, do que alunos nas cidades de Pelotas 
e Rio Grande, e, consequentemente, teriam uma percepção e uma produção mais acurada do que alunos mais distantes da fronteira.

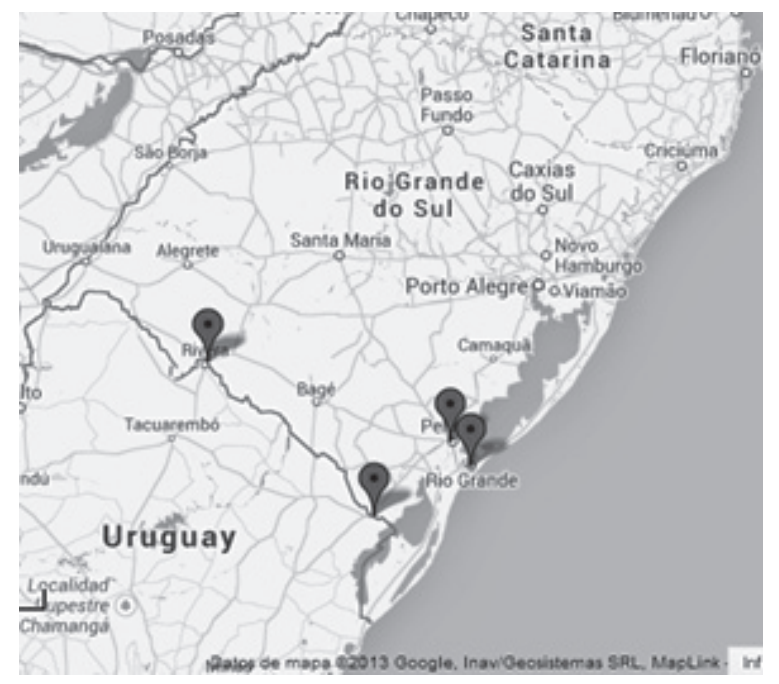

Figura 2 - Mapa com as cidades de coleta de dados. Fonte: Google Maps

\subsection{Procedimentos e instrumentos}

Nesta pesquisa, dois instrumentos foram elaborados para a coleta de dados: 1) um teste de identificação, para avaliar a percepção dos participantes; e 2) uma tarefa de leitura de frases, para coleta de dados de produção.

\subsubsection{Teste de identificação}

Neste estudo, foi usado o programa TP (Teste/Treinamento de Percepção), que é um aplicativo gratuito desenvolvido para a realização de experimentos de Percepção da Fala (RAUBER et al., 2012; SANTOS et al., 2013).

Para a elaboração do teste de identificação, foram selecionadas 76 palavras-alvo dissílabas em espanhol, contendo as vogais [e] (36) e [o] (40) em posição tônica (e.g., mesa, codo). Após a seleção de palavras, foram gravados os estímulos naturais com três locutores falantes nativos de E/L1 (sendo dois homens e uma mulher, com estudo superior, nascidos e residentes em Montevidéu) através da leitura de frases-veículo, como "Diga X una vez", onde " $X$ " representa as palavras selecionadas para o corpus (e.g. "Diga mesa una vez", "Diga codo una vez"). 
No teste de identificação, foram inseridos 240 estímulos no total, permitindo três repetições e uma correção com o botão "Oops"; este se refere à função de "corrigir" a última resposta assinalada, tendo a possibilidade de voltar à questão anterior e refazê-la. O teste foi aplicado em um computador portátil e os estímulos foram ouvidos através de fones de ouvido.

\subsubsection{Teste de produção}

A produção das vogais dos grupos de controle (PB/L1 e E/L1) foi obtida através da leitura de frases apresentadas via o programa PowerPoint (seguindo a metodologia proposta por ESCUDERO et al., 2009; CHLÁDKOVÁ; ESCUDERO; BOERSMA, 2011). Foram utilizadas pseudopalavras, listadas no Quadro 3 do Anexo 1, que continham a seguinte estrutura: CV1CV2, onde "C" são as consoantes /p/, /t/, /k/, /f/, /s/, "V1" são as vogais $/ \mathrm{i}, \mathrm{e}, \mathrm{a}, \mathrm{o}$, u/ e "V2" são as vogais /e, o/. Assim, a frase-veículo lida em espanhol era En CVCe y CVCo tenemos V (e.g., "En pepe y pepo tenemos e") e em português Em CVCe e CVCo temos V (e.g., "Em pepe e pepo temos e"). Para a coleta de dados de produção do grupo E/L2, a frase-veículo "Diga X otra vez" foi apresentada de forma aleatorizada na tela de um computador e continha as palavras usadas no teste de percepção.

As gravações das frases foram realizadas com gravador digital portátil Edirol -Roland R09 e com microfone unidirecional Sony, com taxa de frequência amostral de $44100 \mathrm{~Hz}$. Após as gravações, a taxa de amostragem foi reduzida para $22050 \mathrm{~Hz}$ apenas para poupar espaço na memória do computador. As vogais foram segmentadas com auxílio do programa Praat (BOERSMA; WEENINK, 2011). As análises estatísticas dos dados de percepção e de produção foram realizadas por meio do programa IBM SPSS 17.0.

Com base na metodologia acima descrita, na próxima seção serão apresentados os resultados referentes aos testes de percepção e de produção.

\section{Resultados}

Esta seção encontra-se estruturada em duas partes principais: resultados de percepção e resultados de produção, com suas respectivas análises e discussões.

\subsection{Resultados de percepção}

Esta seção busca responder à primeira questão de pesquisa, que trata da percepção das participantes: "Q1- Como será o desempenho dos falantes 
do PB aprendizes de Espanhol/L2 nos grupos zona de fronteira direta e fronteira indireta, quanto à percepção das vogais-alvo?"

Quanto ao percentual geral de identificação correta no teste de identificação, o grupo de fronteira indireta obteve o maior percentual médio, com $83,58 \%(\mathrm{DP}=13,51)$, enquanto que o grupo fronteira direta obteve um percentual de 78,23\% ( $\mathrm{DP}=15,09)$. O teste estatístico de Mann-Whitney revelou não haver diferenças significativas entre os percentuais médios de ambos os grupos $(\mathrm{Z}=-1,146, \mathrm{p}=0,252)$.

A Figura 3 reporta os percentuais totais de identificação correta por vogais, com o desvio padrão $(\mathrm{DP})$ entre parênteses. $\mathrm{O}$ percentual médio de identificação correta para a vogal [e] foi de $84,02 \%(\mathrm{DP}=14,88)$ para o grupo fronteira indireta e de $79,35 \%(\mathrm{DP}=18,87)$ para o grupo fronteira direta, resultando em diferenças não significativas estatisticamente $(Z=-, 746, p=0,455)$. O percentual médio de identificação correta para a vogal [o] no grupo fronteira indireta foi de $83,60 \%(\mathrm{DP}=12,44)$ e para o grupo fronteira direta de $77,54 \%(\mathrm{DP}=15,04)$, também não se configurando uma diferença significativa $(Z=-1,105, p=0,269)$.

\begin{tabular}{|l|c|c|}
\hline Zona de Fronteira & {$[\mathbf{e}] \%$ (DP) } & {$[\mathbf{o}] \%(\mathrm{DP})$} \\
\hline Direta & $79,35(18,87)$ & $77,54(15,04)$ \\
\hline Indireta & $84,02(14,88)$ & $83,60(12,44)$ \\
\hline
\end{tabular}

Figura 3 - Percentual médio de identificação correta das vogais [e] e [o] nos grupos fronteira direta e fronteira indireta

Em termos percentuais, a diferença entre os grupos fronteira direta e fronteira indireta não é acentuada (cerca de 5\%), porém o grupo fronteira indireta obteve índices de identificação correta ligeiramente maiores no teste de percepção aplicado do que o grupo de fronteira direta. Esperava-se que o grupo fronteira direta obtivesse os maiores índices perceptuais de acerto, corroborando a suposição/hipótese de que informantes de zona de fronteira direta teriam uma percepção mais acurada do que os informantes de zona de fronteira indireta e, como consequência, teriam porcentagens médias superiores, porém os resultados foram ao contrário do esperado. Uma possível explicação pode ser o fato de todas as informantes analisadas serem alunas em final do Curso de Graduação em Letras/Habilitação em Espanhol e, independentemente do local de residência, fatores individuais como a aptidão ou mesmo a motivação do grupo fronteira indireta em se dedicar ao curso podem ter sido mais influentes do que a provável exposição mais frequente à língua espanhola do grupo fronteira direta. 
Como se pode evidenciar através dos testes estatísticos aplicados, os dados de percepção das informantes por zona de fronteira (direta e indireta) apresentam diferenças em termos percentuais, porém, não significativas estatisticamente. Uma vez que se verifica certa homogeneidade no conjunto de dados de percepção, cabe, então, descrever e correlacioná-los com os dados de produção das mesmas informantes.

\subsection{Resultados de produção}

Esta seção visa a responder à segunda pergunta de pesquisa: "Q2-As medições formânticas e de duração das vogais médias do espanhol produzidas por falantes do PB apresentarão variação em relação à zona de fronteira direta e fronteira indireta?"

Quanto às análises por zona de fronteira, na Figura 4 pode-se verificar os valores muito próximos de F1 e F2 das vogais [e] e [o] nos grupos analisados. Quanto às médias de F1 das vogais alvo, o Teste T para Amostras Independentes revelou não haver diferenças significativas entre os valores médios de $\mathrm{F} 1$ entre as informantes da fronteira direta e da fronteira indireta: vogal $[\mathrm{e}](\mathrm{t}(30)=0,406, \mathrm{p}=0,69)$ e vogal $[\mathrm{o}](\mathrm{t}(30)=1,497, \mathrm{p}=0,145)$.

\begin{tabular}{|c|c|c|c|}
\hline Zona de Fronteira & Valores $\mathbf{H z}$ & {$[\mathbf{e}]$} & {$[\mathbf{0}]$} \\
\hline Direta & F1 & $493(18,03)$ & $537(10,18)$ \\
\hline Indireta & F1 & $492(6,29)$ & $532(8,39)$ \\
\hline Direta & F2 & $2.245(0,15)$ & $1.008(0,70)$ \\
\hline Indireta & F2 & $2.136(13,28)$ & $1.079(0,38)$ \\
\hline
\end{tabular}

Figura 4 - Valores médios das medições de F1(Hz) de informantes do sexo feminino (F), com Desvio Padrão (DP) entre parênteses

Quanto ao F2, os resultados do teste de Mann-Whitney revelam que há diferença marginalmente significativa de valores de F2 da vogal [e], indicando que as informantes da fronteira direta apresentam média de F2 levemente superior às da fronteira indireta $(\mathrm{Z}=-1,94, \mathrm{p}=0,52)$. Quanto ao $\mathrm{F} 2$ da vogal [o], as informantes da fronteira indireta apresentaram uma média significativamente maior de $\mathrm{F} 2(1.079 \mathrm{~Hz}, \mathrm{DP}=0,38)$ que as informantes da fronteira direta $(1.008 \mathrm{~Hz}, \mathrm{DP}=0,70, \mathrm{t}(30)=-3,527, \mathrm{p}=0,001)$.

Quanto à duração das vogais, não foram encontradas diferenças significativas entre os grupos: vogal $[\mathrm{e}](\mathrm{t}(30)=0,398, \mathrm{p}=0,694)$ e vogal [o] (t $(30)=0,142, \mathrm{p}=0,888)$. 


\begin{tabular}{|c|c|c|}
\hline Zona de Fronteira & {$[\mathrm{e}]$} & {$[\mathbf{0}]$} \\
\hline Direta & $147(0,02)$ & $154(0,02)$ \\
\hline Indireta & $144(0,02)$ & $152(0,02)$ \\
\hline
\end{tabular}

Figura 6 - Valores médios das medições de Duração (ms), com Desvio Padrão (DP) entre parênteses das informantes de E/L2

Assim, a Figura 7 representa os valores médios de F1 e F2 das vogais [e] e [o] para os grupos fronteira direta (preto) e fronteira indireta (cinza), pertencentes às cidades analisadas.

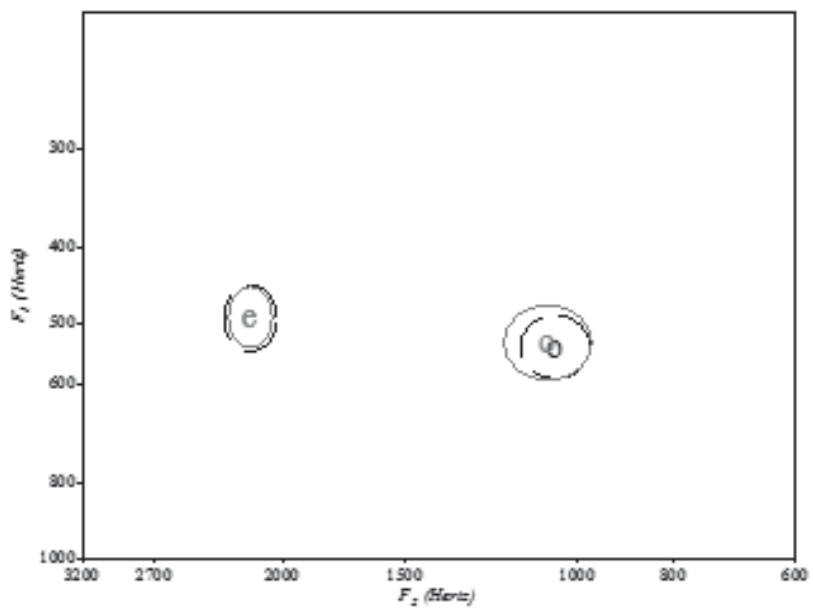

Figura 7- Valores médios de F1 e F2 (em Hz, normalizados), por zona de fronteira

Na Figura 7, pode-se verificar que as produções da vogal [e] em ambos os grupos tiveram a mesma média e praticamente o mesmo campo de dispersão. Quanto à vogal [o], pode-se perceber que as produções diferem um pouco quanto à média (não estaticamente) e quanto ao campo de dispersão, sendo que as informantes da fronteira direta apresentaram um F1 mais baixo do que as da fronteira indireta.

Visto que não houve diferenças significativas, principalmente, quanto aos valores de $\mathrm{F} 1$ entre os grupos fronteira direita e fronteira indireta, considerou-se pertinente reagrupar as 32 informantes em um único grupo, denominado Espanhol/L2 (E/L2), e buscou-se complementar as análises realizadas, acrescentando a comparação das propriedades vocálicas (duração, F1 e F2) das vogais [e] e [o] entre os grupos: $\mathrm{E} / \mathrm{L} 1$ (n=8), PB/L1 (n=8) e E/L2 $(n=32)$. 
Assim, a Figura 8 resume os valores médios de duração, F1 e F2 das vogais [e] e [o] produzidas pelos informantes dos três grupos analisados, E/L1, PB/L1, E/L2, com desvio padrão (DP) entre parênteses.

\begin{tabular}{|c|c|c|c|}
\hline Valores & \multicolumn{3}{|c|}{ Grupos } \\
\hline & E/L1 & PB/L1 & E/L2 \\
\hline Duração [e] & $106(0,01)$ & $136(0,01)$ & $147(0,02)$ \\
\hline Duração [o] & $111(0,01)$ & $136(0,01)$ & $154(0,02)$ \\
\hline F1 [e] & $472(26,46)$ & $441(20,01)$ & $492(6,29)$ \\
\hline F1 [o] & $557(37,01)$ & $489(32,61)$ & $537(10,18)$ \\
\hline F2 [e] & $2270(0,12)$ & $2309(0,20)$ & $2245(0,15)$ \\
\hline F2 [o] & $1010(38,29)$ & $883(90,46)$ & $1008(0,70)$ \\
\hline
\end{tabular}

Figura 8 - Valores médios de duração (ms), F1 (Hz) e F2 Hz) das vogais [e] e [o] nos grupos E/L1, PB/ L1 e E/L2, com desvio padrão (DP) entre parênteses

Os resultados do Teste ANOVA Unifatorial revelam que há diferenças significativas quanto à duração da vogal $[e](F(2,45)=10,500, \mathrm{p}<0,001) \mathrm{e}$ da vogal $[o](F(2,45)=15,530, p<0,001)$ entre os grupos. Esses resultados mostram que a duração da vogal [e] é significativamente menor no grupo $\mathrm{E} / \mathrm{L} 1$ do que no grupo E/L2 ( $<<0,001)$, sendo que os demais grupos não se distinguem de forma estatisticamente significativa (E/L1 versus PBL1: $\mathrm{p}=0,032$ e E/L2 versus PBL1: $\mathrm{p}=0,714)$. Quanto à duração da vogal [o] nos três grupos contrastados, os resultados do teste mostram que a duração de [o] é também significativamente menor no grupo E/L1 do que no grupo $\mathrm{E} / \mathrm{L} 2(\mathrm{p}<0,001)$, sendo que os demais grupos não se distinguem de forma estatisticamente significativa (E/L1 versus $\mathrm{PBL} 1: \mathrm{p}=0,046$ e $\mathrm{E} / \mathrm{L} 2$ versus PBL1: $\mathrm{p}=0,88)$. Assim, conclui-se que, quanto à duração de vogais médias [e] e [o], o grupo E/L2 apresenta valores de duração próximos aos valores do $\mathrm{PB} / \mathrm{L} 1$ (a sua língua materna) e os valores do grupo de E/L1 são significativamente menores que os dos outros dois grupos.

Quanto às frequências formânticas, os resultados do Teste ANOVA Unifatorial revelam que há diferenças significativas quanto aos valores de $\mathrm{F} 1$ para a vogal $[\mathrm{e}](\mathrm{F}(2,45)=30,812, \mathrm{p}<0,001)$ e para a vogal $[\mathrm{o}](\mathrm{F}(2,45)=22,743$, $\mathrm{p}<0,001)$ entre os grupos. Os resultados revelam que a média dos valores de F1 para a vogal [e] do grupo $\mathrm{E} / \mathrm{L} 2(493 \mathrm{~Hz}, \mathrm{DP}=13,32)$ foi significativamente maior do que a média do grupo $\mathrm{E} / \mathrm{L} 1(472 \mathrm{~Hz}, \mathrm{DP}=26,46, \mathrm{p}=0,008)$ e que a do grupo PB/L1(441 Hz, DP=20,01, p<0,001), de modo que a vogal [e] E/L2 
esteja mais distante, mais baixa, de PB/L1 do que de E/L1. Quanto ao F1 da vogal [o], os resultados do Teste ANOVA Unifatorial revelam que a média do grupo $\mathrm{PB} / \mathrm{L} 1(489 \mathrm{~Hz}, \mathrm{DP}=32,61)$ foi significativamente menor do que a dos grupos $\mathrm{E} / \mathrm{L} 2(534 \mathrm{~Hz}, \mathrm{DP}=9,51)(\mathrm{p}<0,001)$ e $\mathrm{E} / \mathrm{L} 1(557 \mathrm{~Hz}, \mathrm{DP}=37,01)$ $(\mathrm{p}<0,001)$, indicando que a vogal [o] do PB é mais alta do que as vogais produzidas nos demais grupos. As diferenças de médias de F1 nos grupos E/L1 e E/L2 não foram estatisticamente significativas $(Z=-1,658, p=0,097)$. Esses resultados indicam que o grupo de E/L2 produziu as vogais [e] e [o] com altura mais próxima à $\mathrm{L} 2$ do que à própria L1.

Quanto aos valores de F2 da vogal [e], os três grupos contrastados não apresentaram diferenças significativas estatisticamente $\left(\chi^{2}(2)=2,134\right.$, $\mathrm{p}=$ n.s.), ou seja, o grau de avanço do dorso da língua é semelhante. Porém, quanto aos valores de F2 da vogal [o], há diferenças significativas que evidenciam que a vogal [o] $\mathrm{PB} / \mathrm{L} 1$ é mais posterior $(\mathrm{Z}=-2,941, \mathrm{p}=0,003)$ do que [o] E/L1 e [o] E/L2, e estes dois grupos não se diferenciam estatisticamente entre si quanto ao grau de recuo do dorso da língua.

Visto que as vogais [e] e [o] produzidas pelas informantes do grupo E/L2 diferiram significativamente das vogais [e] e [o] do E/L1, buscou-se fazer comparação destas com as vogais $[\varepsilon]$ e [0] do $\mathrm{PB}$, respectivamente. Nesta etapa de análises, focalizaram-se os dados referentes aos valores de $\mathrm{F} 1(\varepsilon=621(\mathrm{DP}=45,18), \mathrm{\jmath}=697(\mathrm{DP}=64,04))$, já que as diferenças de médias de F2 não se mostraram significativas na maioria das análises estatísticas.

Os resultados do Teste $\mathrm{T}$ para Amostras Independentes revelam que a média de F1 da vogal [e] E/L2 diferiu significativamente da média da vogal [ع] PB/L1 $(\mathrm{t}(7)=-7,758, \mathrm{p}<0,001)$, como também da vogal [e] do PBL1 $(\mathrm{t}(7)=6,881, \mathrm{p}=0,000)$. Assim, evidencia-se que a vogal [e] $\mathrm{E} / \mathrm{L} 2$ é significativamente mais alta do que a vogal $[\varepsilon] \mathrm{PB} / \mathrm{L} 1$. Dessa forma, entende-se que as produções da vogal [e] E/L2 são desviantes estatisticamente quanto ao F1 de [e] E/L1, visto que as estudantes ainda não produzem a vogal [e] com a mesma altura que a vogal [e] produzida pelas nativas de espanhol.

Em relação ao F1 de [o], a vogal [o] E/L2 diferiu apenas $23 \mathrm{~Hz}$ de [o] E/L1, $45 \mathrm{~Hz}$ de [o] PB/L1 e $163 \mathrm{~Hz}$ de [0] PB/L1. Novamente, os resultados do Teste T para Amostras Independentes revelam que a média de F1 da vogal [o] E/L2 difere significativamente da média de F1 de [o] PB/L1 $(\mathrm{t}(7)=4,047, \mathrm{p}=0,005)$ e de $[0] \mathrm{PB} / \mathrm{L} 1(\mathrm{t}(7)=-7,178, \mathrm{p}<0,001)$.

Assim, em termos de altura, a vogal [e] E/L2 está significativamente distante das vogais $[e, \varepsilon]$ do $\mathrm{PB} / \mathrm{L} 1$; porém, embora significativamente mais baixa, ainda se aproxima mais de [e] E/L1 do que de [e] PB/L1. Os dados mostram que as falantes de E/L2 parecem reorganizar o espaço acústico 
das vogais anteriores para acomodar a categoria vocálica [e] do espanhol, aproximando-se de [e] E/L1 quanto ao avanço do dorso da língua, mas ainda não se aproximando da altura de [e] E/L1 de forma semelhante às nativas do espanhol. Já no espaço acústico das vogais posteriores, [o] E/L2 se assemelha a E/L1 e se distancia estatisticamente de [o, o] do $\mathrm{PB} / \mathrm{L} 1$, indicando que [o] é produzido com altura e recuo do dorso da língua de forma semelhante à nativa do espanhol. Em outras palavras, os dados indicam que a principal pista acústica utilizada pelos falantes de E/ L2 para a produção das vogais médias anteriores parece ser o F1 e para as vogais médias posteriores, o F2.

$\mathrm{Na}$ subseção a seguir, serão apresentadas as considerações finais deste estudo, que investigou a percepção e a produção das vogais médias do E/L2, considerando-se a zona de fronteira.

\section{Considerações finais}

Este estudo teve como motivação a dificuldade dos falantes do $\mathrm{PB}$ em produzir as vogais médias do espanhol, registrada em pesquisas impressionistas (HOYOS-ANDRADE, 1978; MASIP, 1995,1999) e manuais de ensino de E/L2, em geral. Em pesquisas anteriores (BAZZAN, 2005; MIGNONI, 1999) que investigaram as dificuldades dos falantes do $\mathrm{PB}$ com a produção das vogais médias [e, o] do espanhol, ficou evidenciado que os alunos falantes do PB tendiam a realizar estes sons como as vogais médias-baixas $[\varepsilon, \circ]$, respectivamente, da L1. Entretanto, esses estudos não contavam com análises acústicas de dados, nem com a relação entre dados de produção e percepção.

A percepção e a produção das vogais-alvo foram testadas com instrumentos diferentes, bem como os resultados foram tabulados de forma específica: os resultados do teste de percepção, referente à identificação correta das vogais $[\mathrm{e}, \mathrm{o}]$, foram transformados em médias percentuais de acerto; por sua vez, os dados de produção foram medidos em milissegundos (duração) ou Hertz (F1 e F2). Devido à natureza de cada conjunto de resultados, nenhum teste de correlação foi aplicado para avaliar estatisticamente a relação de desempenho dos participantes nos testes de percepção e produção. No entanto, algumas observações podem ser feitas.

Quanto aos resultados de percepção por zona de fronteira, esperava-se que o grupo fronteira direta obtivesse os maiores percentuais de identificação correta no teste de percepção; no entanto, os resultados foram contrários ao esperado, e observou-se que os percentuais médios da fronteira 
indireta foram levemente maiores do que os de fronteira direta. Porém, novamente os resultados dos testes estatísticos aplicados revelaram não haver diferenças significativas entre os grupos quanto ao percentual de acertos por vogal [e] e [o].

Após a aplicação de testes estatísticos, evidenciou-se que os grupos fronteira direita e fronteira indireta não diferem quanto à duração, ao F1 e ao F2 das vogais médias [e] e [o] do espanhol, ou seja, todas as informantes produzem as vogais com duração e altura e avanço/recuo da língua semelhantes.

De acordo com os resultados obtidos neste estudo, pode-se concluir que, quanto à percepção e produção das vogais médias [e] e [o], os falantes de E/L2 não diferem significativamente entre si quanto à zona de fronteira, porém diferem significativamente dos nativos do E/L1 e do PB/L1. A comparação dos resultados do grupo E/L2 com os dos grupos E/L1 e $\mathrm{PB} / \mathrm{L} 1$ revelam um importante achado quanto aos dados de produção de [e] e [o] das falantes de E/L2: estas informantes parecem ter criado categorias vocálicas intermediárias entre as vogais da L1 e da L2. Ao verificar a existência de categorias vocálicas intermediárias entre L1 e L2, permite-se concluir que as altas porcentagens de percepção correta estão relacionadas à produção de categorias vocálicas que se distanciam de valores da L1, mas não chegam a ser significativamente próximas à L2.

Apesar das limitações deste estudo, considera-se que os resultados possuem relevância teórica e prática para uma possível aplicação na área de Aquisição da Fonética e Fonologia de L2. Segundo Gil Fernández (2007), as principais dificuldades na pronúncia das vogais do E/L2 têm origem no sistema fonológico da L1, e podem ser de natureza fonológica, fonética ou distribucional, como por exemplo: confusão e alteração de timbres vocálicos, nasalização excessiva de vogais e realização de voga is com excesso de abertura.

Sabe-se que outros aspectos são importantes na produção de uma L2, como, por exemplo, aspectos segmentais de consoantes e aspectos suprassegmentais que envolvem ritmo e entoação; porém, entende-se que este estudo é uma contribuição que pode abrir caminhos para práticas metodológicas no ensino da fonética e fonologia de L2 que enfatizem a prática da percepção como tão importante quanto à produção de sons.

\section{BIBLIOGRAFIA}

BAZZAN, Maristela Andréa Teichmann. As vogais médias na interfonologia português-espanhol. Dissertação (Mestrado em Linguística Aplicada), Universidade Católica de Pelotas, Pelotas, 2005. 
BEST, Catherine; STRANGE, Winifred. Effects of Phonological and Phonetic Factors on CrossLanguage Perception of Approximants. Haskins Laoratoriu Status Report on Speech Resarch, v. 109/110, p. 89-108, 1992. Disponível em: http://www.haskins.yale.edu/sr/SR109/SR109_07.pdf. Acessado em Ago, 2013.

BEST, Catherine; TYLER, Michael. Nonnative and second-language speech perception: commonalities and complementarities. In BOHN, O.S.; MUNRO, M.(Eds.). Language Experience in second language speech learning: in honor of James Emil Flege. Amsterdam/Philadelphia: John Benjamins Publishing Company. 2007. p. 13-34.

BOERSMA, Paul; WEENINK, David. Praat: doing phonetics by computer [Computer Program], versão 5.2.3. 2008. Disponível em: http://www. praat.org, acesso em 12 maio 2011.

BOHN, Ocke-Schwen. Cross-language speech perception in adults: First language transfer doesn't tell it all. In: STRANGE, W. (Ed.). Speech perception and linguistic experience: Issues in cross-language research. Timonium, MD: York Press, 1995. p. 279-304.

BOHN, Ocke-Schwen. ; FLEGE, James Emil. The production of new and similar vowels by adult German learners of English. Studies in Second Language Acquisition, v. 14, p. 131- 158, 1992.

BRADLOW, Ann. A perceptual comparison of the /i/-/e/ and /u/-/o/ contrasts in English and in Spanish: universal and language specific aspects. Phonetica, v. 53, p. 55-85, 1996.

CÂMARA JUNIOR, Joaquim Mattoso. Estrutura da língua portuguesa. 3. ed. Petrópolis: Vozes, 2007 [1970].

ESCUDERO, Paola; BOERSMA, Paul; RAUBER, Andréia Schurt; BION, Ricardo. A cross-dialect acoustic description of vowels: Brazilian and European Portuguese. Journal of the Acoustical Society of America, v. 126, n. 3, p. 379-1393, 2009.

ESCUDERO, Paola. The role of the input in the development of L1 and L2 sound contrasts: Language-specific cue weighting for vowels. Proceedings of the 25th Annual Boston University Conference on Language Development, Sommerville, MA: Cascadilla Press, 2001.

ESCUDERO, Paola. The perception of English vowel contrasts: Acoustic cue reliance in the development of new contrasts. Proceedings of the fourth international symposium on the acquisition of second language speech, New Sounds 2000. Klagenfurt: University of Klagenfurt, 2000. ESPIGA, Jorge. O Contato do português com o espanhol no Sul do Brasil. 2001. Disponível em: http://www.celpcyro.org.br/v4/html/TextoJorgeEspiga.htm 
FLEGE, James Emil. The production of "new" and "similar" phones in a foreign language: evidence for the effect of equivalence classification. Journal of Phonetics, v.15, p. 47-56, 1987.

FLEGE, James Emil. Second language speech learning theory, findings, and problems. In.: STRANGE, Winifred (Ed.). Speech perception and linguistic experience: issues in cross language research. Timonium, MD: York Press, 1995. p. 233-277.

FLEGE, James Emil; BOHN, Ocke-Schwen; JANG, Sunyoung. Effects of experience on non-native speakers production and perception of English vowels. Journal of Phonetics, v. 25, p. 437-470, 1997.

FLEGE, James Emil; MACKAY, Ian; MEADOR, D. Italian speakers perception and production of English vowels. Journal of the Acoustical Society of America, v. 106, n. 5, p. 2973- 2987, 1999.

FLEGE, James Emil; MUNRO, Murray; FOX, Robert Allen. Auditory and categorical effects on cross language vowel perception. Journal of the Acoustical Society of America, v. 95, n. 6, p. 3623-3641, 1994.

GIL FERNÁNDEZ, Juana. Fonética para profesores de español: de la teoría a la práctica. Madrid: Arco Libros, 2007.

HOYOS-ANDRADE, Rafael Eugenio. El vocalismo del español y del portugués: estudio contrastivo fonético-fonológico y sus implicaciones pedagógicas. Lengua y Literatura, v. 7, p. 369-380, 1978.

KLUGE, Denise Cristina. Perception and production of English syllable-final nasals by Brazilian learners. Dissertação (Mestrado em Letras/Inglês e Literatura Correspondente), Universidade Federal de Santa Catarina, Florianópolis, 2004.

KLUGE, Denise Cristina. Brazilian EFL learners' identification of word-final /m-n/: native/nonnative realizations and effect of visual cues. Tese (Doutorado em Letras/Inglês), Universidade Federal de Santa Catarina, Florianópolis, 2009.

KOERICH, R. D. (2002). Perception and production of word-final vowel epenthesis by Brazilian EFL students. Tese (Doutorado em Letras), Universidade Federal de Santa Catarina, Florianópolis, 2002.

MAJOR, R. Phonological similarity, markedness, and rate of 12 acquisition. Studies in Second Language Acquisition, v. 9, n. 1, p. 63-82, 1987. MARTÍNEZ CELDRÁN, Eugenio; FERNÁNDEZ PLANAS, Ana María. Manual de fonética española. Barcelona: Ariel, 2007.

MASIP, Vicente. Dificuldades fonéticas segmentais por estudantes recifenses de espanhol. Tese (Doutorado em Letras), Universidade Federal de Pernambuco, Recife, 1995. 
MASIP, Vicente. Fonética espanhola para brasileiros - síntese. Revista GELNE, ano 1, n. 1, p. 152-158, 1999.

MIGNONI, Rosália Lacerda. A transferência e a aquisição das vogais espanholas /e/ e /o/ em substantivos e adjetivos por falantes universitários brasileiros. Dissertação (Mestrado em Letras), Universidade Federal do rio Grande do Sul, Porto Alegre, 1999.

MORRISON, Geoffrey Stewart. Perception of English /i/ and /I/ by Japanese and Spanish listeners: Longitudinal results. In: MORRISON, G. S.; ZSOLDOS, L. (Eds.). Proceedings of the North West Linguistics Conference, Canada: Simon Fraser University Linguistics Graduate Student Association, 2002. p. 29-48.

NOBRE-OLIVEIRA, Denize. The effects of perceptual training on the learning of English vowels by Brazilian Portuguese speakers. Tese (Doutorado em Letras), Universidade Federal de Santa Catarina, Florianópolis, 2007. PASCA, Maria Alejandra Saraiva. Aspectos da aquisição da vogal oral /a/ em língua espanhola por estudantes de língua portuguesa: a questão da percepção. Dissertação (Mestrado em Letras), Universidade Federal do Rio Grande do Sul, Porto Alegre, 2003.

PISKE, Thorsten; MACKAY, Ian; FLEGE, James Emil. Factors affecting degree of foreign accent in an L2: A review. Journal of Phonetics, v. 29, p. 191-215, 2001.

RAUBER, Andréia Schurt. Production of English initial /s/-clusters by speakers of Brazilian Portuguese and Argentine Spanish. In: BATISTA, B.; WATKINS, M.A. (Eds.). English with a Latin Beat: Studies in Portuguese/ Spanish- English Interphonology. Amsterdam: John Benjamins, 2006. p. 155-167.

RAUBER, Andréia Schurt. Acoustic characteristics of Brazilian English vowels: perception and production results. Saarbrücken: Lambert Academic Publishing, 2010.

RAUBER, Andréia Schurt; RATO, Anabela; SILVA, Ana Lúcia. Percepção e produção de vogais anteriores do inglês por falantes nativos de mandarim. Diacrítica, v. 24, n. 1, p. 5-23, 2010.

RAUBER, Andréia Schurt; RATO, Anabela, KLUGE, Denise Cristina;

SANTOS, Giane Rodrigues dos. TP: testes de percepção/tarefas de treinamento perceptual [aplicativo]. Disponível em: http://www.worken.com. br/tp, acesso em: 11 maio 2012.

RESTREPO, Júlia Crochemore. Percepção e produção de aprendizes brasileiros de francês: o caso das vogais médias anteriores arredondadas. Dissertação (Mestrado em Linguística), Universidade Federal de Santa Catarina, Florianópolis, 2011. 
ROCHET, B. Perception and production of second-language speech sounds by adults. In: STRANGE, Winifred (Ed.). Speech perception and linguistic experience: issues in cross-language research. Timonium, MD: York Press, 1995. p. 379-410.

SANTOS, Giane Rodrigues dos. Percepção e produção das vogais médias do espanhol por falantes do português brasileiro. Tese (Doutorado em Letras), Universidade Católica de Pelotas, Pelotas, 2014.

SANTOS, Giane Rodrigues dos; RAUBER, Andréia Schurt; RATO, Anabela; KLUGE, Denise Cristina; FIGUEIREDO, Marcos Guilherme. TP (v. 3.1): una herramienta para experimentos de percepción. Estudios de Fonética Experimental, v. 22, p. 335-366, 2013.

SILVEIRA, Rosane. The influence of pronunciation instruction on de perception and production of English word-final consonants. Tese (Doutorado em Letras), Universidade Federal de Santa Catarina, Florianópolis, 2004.

Recebido em: 01/12/2014. Aceito em: 03/03/2015. 